\title{
Electronic-state-lifetime interference in the hard-x-ray regime: Argon as a showcase
}

\author{
G. Goldsztejn, ${ }^{1, *}$ R. Püttner, ${ }^{2}$ L. Journel, ${ }^{1,3}$ R. Guillemin, ${ }^{1,3}$ O. Travnikova, ${ }^{1,3}$ R. K. Kushawaha, ${ }^{1}$ B. Cunha de Miranda, ${ }^{1}$ \\ I. Ismail, ${ }^{1}$ D. Céolin, ${ }^{3}$ M. N. Piancastelli, ${ }^{1,4}$ M. Simon,,${ }^{1,3}$ and T. Marchenko ${ }^{1,3, \dagger}$ \\ ${ }^{1}$ Sorbonne Universités, UPMC Université Paris 06, CNRS, UMR 7614, Laboratoire de Chimie Physique-Matière et Rayonnement, \\ F-75005 Paris, France \\ ${ }^{2}$ Fachbereich Physik, Freie Universität Berlin, Arnimallee 14, D-14195 Berlin, Germany \\ ${ }^{3}$ Synchrotron SOLEIL, l'Orme des Merisiers, Saint-Aubin, BP 48, F-91192 Gif-sur-Yvette Cedex, France \\ ${ }^{4}$ Department of Physics and Astronomy, Uppsala University, PO Box 516, SE-751 20 Uppsala, Sweden
}

(Received 8 November 2016; published 30 January 2017)

\begin{abstract}
Electronic-state-lifetime interference is a phenomenon specific for ionization of atoms and molecules in the hard-x-ray regime. Using resonant $K L_{2,3} L_{2,3}$ Auger decay in argon as a showcase, we present a model that allows extracting the interference terms directly from the cross sections of the final electronic states. The analysis provides fundamental information on the excitation and decay processes such as probabilities of various decay paths and the values of the dipole matrix elements for transitions to the excited states. Our results shed light on the interplay between spectator, shake-down, and shake-up processes in the relaxation of deep core-hole states.
\end{abstract}

DOI: 10.1103/PhysRevA.95.012509

\section{INTRODUCTION}

Interference is a key phenomenon in quantum physics, more particularly so in the interaction of light with matter. From Young's experiment [1] to quantum optics experiments where only one photon is emitted [2-4], the observed interference patterns demonstrated that systems could be prepared in a coherent superposition of states. In atomic and molecular physics, interference effects can occur if different excited electronic and/or vibrational states are coherently populated and decay into the same final state.

As an example, coherent excitation of different vibrational states with the natural lifetime broadening of the same order of magnitude as the vibrational spacing leads to the lifetimevibrational interference (LVI) phenomenon. This has been thoroughly studied in the soft-X-ray regime, where several experiments on the $\mathrm{CO}$ molecule at the $\mathrm{C} 1 s \rightarrow \pi^{*}$ resonance [5-9], at the C $1 s \rightarrow$ Rydberg orbitals [10], and at the O $1 s \rightarrow \pi^{*}$ resonance [11] showed evidence of the LVI effects. Later such effects were also observed in the $\mathrm{N}_{2}$ [12] and $\mathrm{O}_{2}$ [13] molecules.

In the hard-X-ray regime, the lifetime broadening of excited states can be on the order of $1 \mathrm{eV}$, which is comparable to the energy spacing between the excited electronic states. Different excited states can therefore be coherently populated and can decay, either through spectator Auger or with involvement of shake processes, into the same final state, which induces electronic-state-lifetime interference (ELI).

Interference phenomena between discrete and continuum electronic states were predicted by Fano a long time ago [14]. Ảberg developed a unified theory that takes into account LVI and ELI effects [15] and Cesar and Ågren elaborated a theoretical description of these phenomena in the case of radiative and nonradiative decays [16]. However, only a few

\footnotetext{
*gildas.goldsztejn@mbi-berlin.de

${ }^{\dagger}$ Corresponding author: tatiana.marchenko@upmc.fr
}

experimental observations of ELI can be found in the literature. Levin et al. performed coincidence measurements between ions and Auger electrons at the $K$ edge of the argon atom [17] and Rubensson et al. observed interference effects between the $1 s^{-1} 3 p^{\prime}$ and $1 s^{-1} 4 p^{\prime}$ resonances in the neon atom [18]. LeBrun et al. measured Auger spectra after resonant excitation or ionization at the $K$ edge in argon with an experimental resolution above $1 \mathrm{eV}$ and observed an asymmetric profile in the $2 p^{-2} 5 p^{+}$cross section due to ELI [19]. Here and in the following we use the notation $n p^{\prime}$ for the excited electronic states and $n p^{+}$for the final ionized states to avoid confusion. In all the studies cited above, a modest experimental resolution did not allow the authors to disentangle the contributions of different intermediate states relaxing into one particular final state.

Recently, high-resolution resonant inelastic x-rayscattering measurements allowed observation of ELI in the core-excited $\mathrm{HCl}$ molecule [20]. However, due to strict selection rules governing the radiative decay, the observed ELI effect was rather weak. The high-resolution hard-X-ray photoemission spectroscopy (HAXPES) end-station installed on the GALAXIES beamline at the French synchrotron SOLEIL enabled us to observe strong ELI effects in resonant Auger spectra at the $K$ edge in argon [21], at the $L$ edge in xenon [22], and at the $\mathrm{Cl} K$ edge in the $\mathrm{HCl}$ and $\mathrm{CH}_{3} \mathrm{Cl}$ molecules [23]. The ELI has been observed in the cross sections of the final states reflecting the direct contributions of different decay channels as well as the interference occurring between them.

In [23] we introduced a model that allows extracting the ELI terms as well as the intensity ratios of the overlapping final states. In the present paper we demonstrate the capacity of this model to provide fundamental information on the excitation and decay processes such as probabilities of different decay channels and the values of the dipole transition matrix elements for the excitation at the $K$ edge in the argon atom.

The article is organized as follows. The experimental setup is described in Sec. II. The model formalism is detailed in Sec. III. We present our data analysis and results in Sec. IV. We summarize and give our conclusions in Sec. V. 


\section{EXPERIMENTAL DETAILS}

The experimental setup was thoroughly described in [24]. Briefly, the measurements were performed using the endstation HAXPES, based on a hemispherical electron analyzer permanently installed on the GALAXIES beamline at the synchrotron SOLEIL [25]. The analyzer has a wide angular acceptance and allows measurements of high-energy electrons (up to $10 \mathrm{keV}$ ) that can be emitted from atoms and molecules ionized with the photons of the GALAXIES beamline in the 2.3-12 keV energy range. The $\mathrm{x}$-ray light from the U20 undulator is linearly polarized in the horizontal plane and monochromatized by a $\mathrm{Si}(111)$ double-crystal monochromator. The lenses of the analyzer are set parallel to the polarization vector. Note that, due to the fixed experimental geometry, the cross sections discussed throughout the paper are not the absolute cross sections (see [22,23,26] for details). The total instrumental resolution of $460 \mathrm{meV}$ [21] includes the contributions of the photon bandwidth $(\approx 400 \mathrm{meV})$, the spectrometer resolution $(\approx 200 \mathrm{meV})$, and the thermal Doppler broadening $(\approx 100 \mathrm{meV})$, since the measurements were performed at room temperature. The $K L_{2,3} L_{2,3}$ Auger spectra were recorded while changing the incident photon energy across the $1 s-n p^{\prime}(n \geqslant 4)$ resonances and up to $1 \mathrm{eV}$ above ionization threshold.

In order to extract the cross sections of the final states as a function of the incident photon energy, we follow the procedure described in [21]. The peaks in the Auger spectra corresponding to the $2 p^{-2} n p^{+}(n \geqslant 4)$ final states were fitted with Voigt functions, which represent a convolution between a Lorentzian function describing the electronic transition to the final state and a Gaussian function taking into account the total instrumental resolution.

\section{SIMULATIONS}

In our recent work [23] we developed a model allowing us to qualitatively describe the effect of ELI in the cross sections of the final states in the Auger spectra of core-excited atoms and molecules. In general, the model describes the interference between multiple excited electronic states and takes into account nuclear dynamics occurring in a dissociative excited molecular state. Here we demonstrate that in the case of the argon atom the model allows extracting quantitative information about the dipole transition matrix elements for the $\mathrm{Ar} 1 s$ excitation and the probabilities of different decay channels. For clarity, here we outline the formalism of the model in a basic case of two discrete electronic excited states.

The cross section $\sigma(\omega)$ of a given final state is described by the Kramers-Heisenberg formula [27]

$$
\sigma(\omega) \propto\left|\sum_{c} \frac{\left\langle\Phi_{f}|\mathcal{Q}| \Phi_{c}\right\rangle\left\langle\Phi_{c}|\mathcal{D}| \Phi_{0}\right\rangle}{\omega-\tau_{c}+i \Gamma_{c} / 2}\right|^{2} \delta\left(\omega-\omega^{\prime}-\tau_{f}, \Gamma_{c}\right),
$$

where $\sum_{c}$ represents the sum over all the intermediate electronic states, $\left\langle\Phi_{c}|\mathcal{D}| \Phi_{0}\right\rangle$ is the dipole matrix element that corresponds to the transition from the ground state described by the wave function $\Phi_{0}$ to the intermediate excited state described by the wave function $\Phi_{c}$, and $\left\langle\Phi_{f}|\mathcal{Q}| \Phi_{c}\right\rangle$ is the
Coulomb matrix element corresponding to the transition from the intermediate to the final state described by the wave function $\Phi_{f}$. Further, $\omega$ and $\omega^{\prime}$ are, respectively, the energies of the incident photon and of the emitted Auger electron, $\tau_{c}$ and $\tau_{f}$ are the energy differences between the ground and the intermediate electronic states and between the ground and the final electronic states, respectively, and $\Gamma_{c}$ is the lifetime broadening of the intermediate state. Finally, $\delta(\omega-$ $\left.\omega^{\prime}-\tau_{f}, \Gamma_{c}\right)$ is a Lorentzian function that takes into account the energy conservation throughout the whole absorption and decay process.

In the case of two discrete intermediate electronic states, the cross section can be rewritten

$$
\sigma(\omega) \propto\left|\frac{K_{1}}{\omega-\tau_{1}+i \Gamma_{1} / 2}+\frac{K_{2}}{\omega-\tau_{2}+i \Gamma_{2} / 2}\right|^{2},
$$

where $K_{c}=\left\langle\Phi_{f}|\mathcal{Q}| \Phi_{c}\right\rangle\left\langle\Phi_{c}|\mathcal{D}| \Phi_{0}\right\rangle$ (c=1 or 2). The righthand side of Eq. (2) can be developed into a sum of two Lorentzian functions $\mathcal{L}$ and a cross term

$$
\begin{aligned}
\sigma(\omega) \propto & \mathcal{L}\left(\Gamma_{1}, \tau_{1}, K_{1}, \omega\right)+\mathcal{L}\left(\Gamma_{2}, \tau_{2}, K_{2}, \omega\right) \\
& +\frac{\left(K_{1} K_{2}^{*}+K_{1}^{*} K_{2}\right)\left[\left(\omega-\tau_{1}\right)\left(\omega-\tau_{2}\right)+\frac{\Gamma_{1} \Gamma_{2}}{4}\right]}{\left[\left(\omega-\tau_{1}\right)^{2}+\frac{\Gamma_{1}^{2}}{4}\right]\left[\left(\omega-\tau_{2}\right)^{2}+\frac{\Gamma_{2}^{2}}{4}\right]}
\end{aligned}
$$

In the case of $K L L$ Auger decay, the core holes created in both the intermediate and the final states can be considered as sufficiently deep to remain unaffected by the presence of an excited electron in a valence shell. This allowed Armen et al. [28] to make an approximation suggesting that the Coulomb matrix element can be decomposed into a constant partial $K L L$ Auger rate amplitude and an overlap integral

$$
\left\langle 1 s^{-1} m p^{\prime}|\mathcal{Q}| 2 p^{-2} n p^{+}\right\rangle=\left\langle 1 s^{-1}|\mathcal{Q}| 2 p^{-2}\right\rangle\left\langle m p^{\prime} \mid n p^{+}\right\rangle .
$$

Here $\Gamma_{K L L}=\left|\left\langle 1 s^{-1}|\mathcal{Q}| 2 p^{-2}\right\rangle\right|^{2}$ is the partial Auger rate for the $K L L$ transition and $\left|\left\langle m p^{\prime} \mid n p^{+}\right\rangle\right|^{2}$ is directly related to the probability of spectator or shake processes.

After introducing Eq. (4) into Eq. (3), we obtain

$$
\begin{aligned}
\sigma(\omega) \propto & \mathcal{L}\left(\Gamma_{1}, \tau_{1}, k_{1}, \omega\right)+\mathcal{L}\left(\Gamma_{2}, \tau_{2}, k_{2}, \omega\right) \\
& +\frac{\left(k_{1} k_{2}^{*}+k_{1}^{*} k_{2}\right)\left[\left(\omega-\tau_{1}\right)\left(\omega-\tau_{2}\right)+\frac{\Gamma_{1} \Gamma_{2}}{4}\right]}{\left[\left(\omega-\tau_{1}\right)^{2}+\frac{\Gamma_{1}^{2}}{4}\right]\left[\left(\omega-\tau_{2}\right)^{2}+\frac{\Gamma_{2}^{2}}{4}\right]} .
\end{aligned}
$$

Here $k_{1,2}=\frac{K_{1,2}}{\sqrt{\Gamma_{K L L}}}=\left\langle 1 s^{-1} m p^{\prime}|\mathcal{D}| \Phi_{0}\right\rangle\left\langle m p^{\prime} \mid n p^{+}\right\rangle$. Note that $K_{1,2}$ and $k_{1,2}$ can be chosen as real parameters without loss of generality [23]; then $k_{1} k_{2}^{*}+k_{1}^{*} k_{2}=2 k_{1} k_{2}$.

In the following we will apply Eq. (5) for fitting the experimental cross sections of the final states $2 p^{-2} n p^{+}$, using $k_{1,2}, \tau_{1,2}$, and $\Gamma_{1,2}$ as fit parameters. Analysis of the fitting results allows obtaining quantitative estimates for the excitation dipole transition matrix elements and for the probabilities of different decay channels. The results of our analysis are presented in the following section.

\section{RESULTS}

Figure 1 presents the results of fitting the experimental cross sections of the final states $2 p^{-2} n p^{+}(4 \leqslant n \leqslant 7)$ plotted as a function of the photon energy relative to the ionization potential (IP). Here the circles are the experimental points 


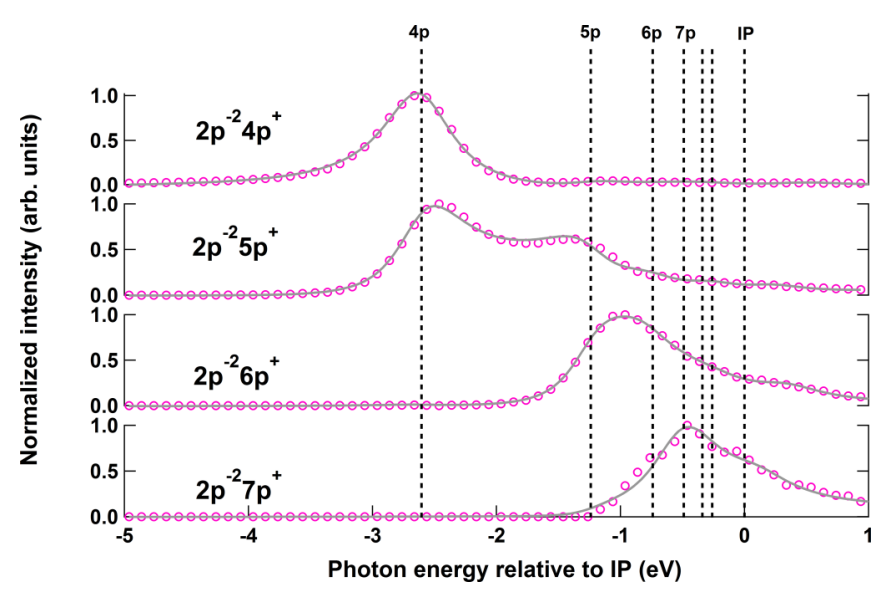

FIG. 1. Cross sections of the final states $2 p^{-2} n p^{+}(4 \leqslant n \leqslant 7)$ plotted as a function of the photon energy relative to the ionization potential. The circles are the experimental points and the lines are the fitting results. The vertical lines indicate the energies of the resonantly excited intermediate states $1 s^{-1} m p^{\prime}(4 \leqslant m \leqslant 9)$.

and the lines are the results of our fits. We assumed the lifetime broadening $\Gamma$ to be constant for all core-excited states $1 s^{-1} m p^{\prime}$. The fitting provides the value $\Gamma=0.7 \mathrm{eV}$, in agreement with [29]. The cross sections of the final states $2 p^{-2} 4 p^{+}, 2 p^{-2} 5 p^{+}, 2 p^{-2} 6 p^{+}$, and $2 p^{-2} 7 p^{+}$are normalized to the dominant peak intensities: $I_{4 p}=4593.5, I_{5 p}=1546.2$, $I_{6 p}=1119.1$, and $I_{7 p}=646.4$, respectively. The intensity values are in arbitrary units and are related to the statistics of our experimental conditions, such as gas pressure and acquisition time.

The energies $E_{m, l}$ of the resonantly excited intermediate states $1 s^{-1} m p^{\prime}(4 \leqslant m \leqslant 9)$ are indicated with vertical lines in Fig. 1 and summarized in Table I. The energy value of the $1 s^{-1} 4 p^{\prime}$ state was determined from the fit of the $2 p^{-2} 4 p^{+}$ cross section, while keeping free the $\tau_{c}$ fitting parameter describing the energy of the resonant transition from the ground to the intermediate state [see Eqs. (1) and (5)]. The determined energy of the $1 s^{-1} 4 p^{\prime}$ state and the known value of the IP (3205.9 eV) allowed calculating the quantum defect $(\delta=1.72)$ and therefore empirically determine the resonant energies of the other $1 s^{-1} m p^{\prime}$ states

$$
E_{m, l}=V_{\text {ion }}-\frac{R_{\infty}}{(m-\delta)^{2}},
$$

where $V_{\text {ion }}$ is the IP and $R_{\infty}$ is the Rydberg constant.

TABLE I. Energies of the resonantly excited intermediate states $1 s^{-1} m p^{\prime}(4 \leqslant m \leqslant 9)$, relative to the IP.

\begin{tabular}{ll}
\hline \hline Electronic state & $E_{m, l}(\mathrm{eV})$ \\
\hline $1 s^{-1} 4 p^{\prime}$ & -2.61 \\
$1 s^{-1} 5 p^{\prime}$ & -1.24 \\
$1 s^{-1} 6 p^{\prime}$ & -0.74 \\
$1 s^{-1} 7 p^{\prime}$ & -0.49 \\
$1 s^{-1} 8 p^{\prime}$ & -0.34 \\
$1 s^{-1} 9 p^{\prime}$ & -0.26 \\
\hline \hline
\end{tabular}

The cross section of the $2 p^{-2} 4 p^{+}$final state shown in Fig. 1 contains one broad peak with the maximum located close to the resonant energy of the $1 s-4 p$ transition, which reflects the dominance of the spectator Auger decay. The absence of any significant asymmetry or shift from the resonant $1 s^{-1} 4 p^{\prime}$ excitation energy indicates the weakness of the ELI effects in this case.

A very different situation can be observed in the cross section of the $2 p^{-2} 5 p^{+}$final state dominated by two intense peaks. One peak is located close to the $1 s^{-1} 5 p^{\prime}$ resonance and corresponds to the spectator Auger decay. The other peak has the maximum near the energy of the $1 s-4 p$ transition and corresponds to a shake-up process, where the excited electron, initially promoted to the $4 p$ orbital, ends up in the $5 p$ orbital in the final state. One can observe that both peaks are strongly asymmetric and their maxima are displaced with respect to the resonant energies, which indicates a strong manifestation of the ELI phenomenon. The reduction of the energy difference between the maxima of the peaks, observed in the cross section of the $2 p^{-2} 5 p^{+}$final state, with respect to the difference between the resonant energies of the $1 s-4 p$ and $1 s-5 p$ transitions, is characteristic for the manifestation of ELI between the spectator Auger decay and the decay channel with involvement of a shake-up process $[23,30]$. Furthermore, a weak contribution near the energy of the $1 s-6 p$ transition in the cross section of the $2 p^{-2} 5 p^{+}$final state corresponds to a shakedown process, where the excited electron, initially promoted to the $6 p$ orbital, ends up in the $5 p$ orbital in the final state.

The cross section of the $2 p^{-2} 6 p^{+}$final state contains a broad asymmetric peak located between the $1 s^{-1} 5 p^{\prime}$ and $1 s^{-1} 6 p^{\prime}$ resonances and a shoulder near the $1 s^{-1} 7 p^{\prime}$ resonance. A detailed analysis of various decay channels contributing to this cross section and the role of the ELI effects are presented below (see Fig. 2). Finally, in the cross section of the $2 p^{-2} 7 p^{+}$final state, one can observe a dominant peak located near the $1 s^{-1} 7 p^{\prime}$ resonance, corresponding to the spectator decay, and several shoulders at lower and higher energies due to contributions from shake-up and shake-down processes, respectively.

The relative amplitudes of different decay channels contributing to each of the final states have been obtained as a result of our fitting procedure using Eq. (5). The corresponding fit parameters $k=\left\langle\Phi_{0}|\mathcal{D}| 1 s^{-1} m p^{\prime}\right\rangle\left\langle m p^{\prime} \mid n p^{+}\right\rangle$are summarized in Table II, where the relative amplitudes are normalized to the peak intensity of the dominant contribution $I_{n p}(4 \leqslant n \leqslant 7)$ as described above in relation to Fig. 1. Analysis of the results presented in Table II allows us to make two observations.

First, one can observe that the amplitudes of the decay channels involving shake-up processes have a sign opposite to that of the spectator decay channels and the decays accompanied by shake-down processes. Although the physical interpretation requires further theoretical analysis, we assume that this observation may be related to the difference in the size of the orbitals occupied by the excited electron in the intermediate and the final states.

The observed trend is directly related to the relative displacements of the peaks in the cross sections of the final states due to the ELI effect. The direction of the relative displacement depends on the sign of the interference cross term in Eq. (5), 


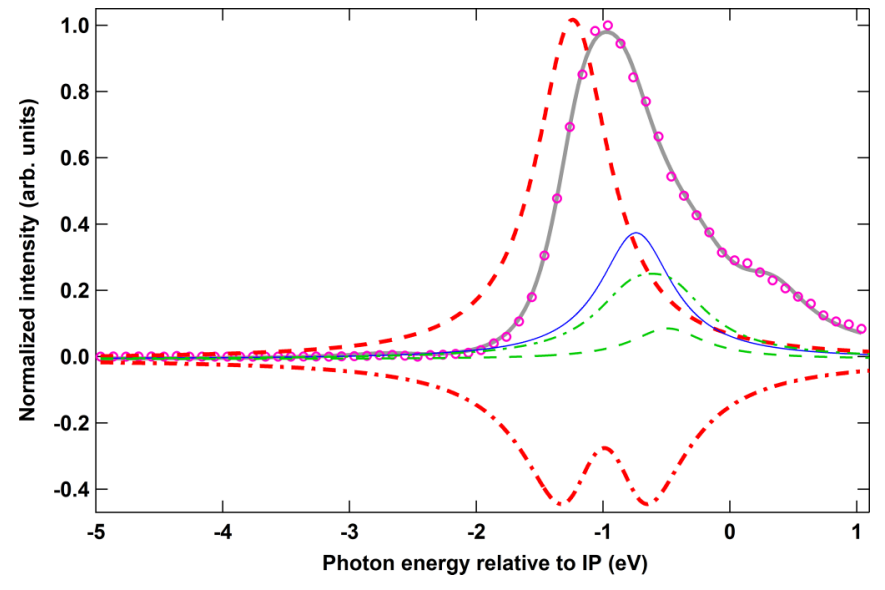

FIG. 2. Cross section of the $2 p^{-2} 6 p^{+}$final state plotted as a function of the photon energy relative to the ionization potential. The circles are the experimental points, the thick solid gray line is the fitting result. The thin solid blue, thick dashed red, and thin dashed green lines show the contributions of the spectator decay, the decay accompanied by a shake-up process from the $5 p$ orbital, and the decay involving a shake-down process from the $7 p$ orbital, respectively. The interference cross terms between the $1 s^{-1} 6 p^{\prime}$ and $1 s^{-1} 5 p^{\prime}$ electronic states and between the $1 s^{-1} 6 p^{\prime}$ and $1 s^{-1} 7 p^{\prime}$ electronic states are shown with thick dash-dotted red and thin dash-dotted green lines, respectively.

which is determined by the product $k_{1} k_{2}$ of the relative amplitudes of the interfering decay channels. As demonstrated in Fig. 1, the maxima of the peaks corresponding to the spectator Auger decay and the decay channel with involvement of a shake-up process are displaced towards each other due to the ELI effect. In this case the product of the relative amplitudes of the interfering channels and hence the interference cross term have a negative sign. One can show that in the case of

TABLE II. Relative amplitudes of different decay channels contributing to the cross sections of the $2 p^{-2} n p^{+}$final states, normalized to the intensity of the dominant peak.

\begin{tabular}{lll}
\hline \hline Final state & \multicolumn{1}{c}{ Fit parameter $k$} & Decay type \\
\hline & $\left\langle\Phi_{0}|\mathcal{D}| 1 s^{-1} 4 p^{\prime}\right\rangle\left\langle 4 p^{\prime} \mid 4 p^{+}\right\rangle=1.00$ & spectator \\
$2 p^{-2} 4 p^{+}$ & $\left\langle\Phi_{0}|\mathcal{D}| 1 s^{-1} 5 p^{\prime}\right\rangle\left\langle 5 p^{\prime} \mid 4 p^{+}\right\rangle=0.15 \pm 0.01$ & shake-down \\
& $\left\langle\Phi_{0}|\mathcal{D}| 1 s^{-1} 6 p^{\prime}\right\rangle\left\langle 6 p^{\prime} \mid 4 p^{+}\right\rangle=0.07 \pm 0.03$ & shake-down \\
& $\left\langle\Phi_{0}|\mathcal{D}| 1 s^{-1} 7 p^{\prime}\right\rangle\left\langle 7 p^{\prime} \mid 4 p^{+}\right\rangle=0.04 \pm 0.03$ & shake-down \\
& $\left\langle\Phi_{0}|\mathcal{D}| 1 s^{-1} 4 p^{\prime}\right\rangle\left\langle 4 p^{\prime} \mid 5 p^{+}\right\rangle=-1.00$ & shake-up \\
& $\left\langle\Phi_{0}|\mathcal{D}| 1 s^{-1} 5 p^{\prime}\right\rangle\left\langle 5 p^{\prime} \mid 5 p^{+}\right\rangle=0.49 \pm 0.02$ & spectator \\
$2 p^{-2} 5 p^{+}$ & $\left\langle\Phi_{0}|\mathcal{D}| 1 s^{-1} 6 p^{\prime}\right\rangle\left\langle 6 p^{\prime} \mid 5 p^{+}\right\rangle=0.22 \pm 0.04$ & shake-down \\
& $\left\langle\Phi_{0}|\mathcal{D}| 1 s^{-1} 7 p^{\prime}\right\rangle\left\langle 7 p^{\prime} \mid 5 p^{+}\right\rangle=0.15 \pm 0.10$ & shake-down \\
& $\left\langle\Phi_{0}|\mathcal{D}| 1 s^{-1} 4 p^{\prime}\right\rangle\left\langle 4 p^{\prime} \mid 6 p^{+}\right\rangle=-0.10 \pm 0.03$ & shake-up \\
$2 p^{-2} 6 p^{+}$ & $\left\langle\Phi_{0}|\mathcal{D}| 1 s^{-1} 5 p^{\prime}\right\rangle\left\langle 5 p^{\prime} \mid 6 p^{+}\right\rangle=-1.00$ & shake-up \\
& $\left\langle\Phi_{0}|\mathcal{D}| 1 s^{-1} 6 p^{\prime}\right\rangle\left\langle 6 p^{\prime} \mid 6 p^{+}\right\rangle=0.57 \pm 0.04$ & spectator \\
& $\left\langle\Phi_{0}|\mathcal{D}| 1 s^{-1} 7 p^{\prime}\right\rangle\left\langle 7 p^{\prime} \mid 6 p^{+}\right\rangle=0.32 \pm 0.04$ & shake-down \\
& $\left\langle\Phi_{0}|\mathcal{D}| 1 s^{-1} 4 p^{\prime}\right\rangle\left\langle 4 p^{\prime} \mid 7 p^{+}\right\rangle=0.00$ & shake-up \\
& $\left\langle\Phi_{0}|\mathcal{D}| 1 s^{-1} 5 p^{\prime}\right\rangle\left\langle 5 p^{\prime} \mid 7 p^{+}\right\rangle=-0.31 \pm 0.08$ & shake-up \\
& $\left\langle\Phi_{0}|\mathcal{D}| 1 s^{-1} 6 p^{\prime}\right\rangle\left\langle 6 p^{\prime} \mid 7 p^{+}\right\rangle=-0.15 \pm 0.11$ & shake-up \\
& $\left\langle\Phi_{0}|\mathcal{D}| 1 s^{-1} 7 p^{\prime}\right\rangle\left\langle 7 p^{\prime} \mid 7 p^{+}\right\rangle=1.00$ & spectator \\
\hline \hline
\end{tabular}

interfering spectator decay and the decay channel involving a shake-down process, the interference cross term is positive and the maxima of the peaks are shifted away from each other.

A second observation resulting from the analysis of Table II indicates the dominance of the decay channel accompanied by a shake-up process in the cross sections of the $2 p^{-2} 5 p^{+}$and the $2 p^{-2} 6 p^{+}$final states. This effect may be related to the contraction of the Rydberg orbitals upon the $K L L$ Auger decay. Namely, the Auger decay leads to a sudden enhancement of the effective charge experienced by the electrons in the outer orbitals, causing the orbitals' contraction towards the nucleus. In this case, an overlap between the intermediate $1 s^{-1} 5 p^{\prime}$ and the final $2 p^{-2} 6 p^{+}$states may become larger than an overlap between the intermediate $1 s^{-1} 6 p^{\prime}$ and the final $2 p^{-2} 6 p^{+}$states, thus favoring decay through a shake-up process over the spectator decay.

The roles of different decay channels and the influence of the ELI are illustrated in the cross section of the $2 p^{-2} 6 p^{+}$ final state presented in Fig. 2. The dominant contribution is the decay channel involving a shake-up process, where the excited electron, initially promoted to the $5 p$ orbital, results in the $6 p$ orbital in the final state. A strong interference between the spectator decay (thin solid blue line) and the decay involving a shake-up process (thick dashed red line) becomes evident through a corresponding interference cross term (thick dash-dotted red line). Due to the negative sign of the cross term, the peaks of the interfering contributions shift towards each other, merging into a single broad asymmetric peak in the cross section of the final state (thick solid gray line) with a maximum located at the relative energy around $-1 \mathrm{eV}$, between the $1 s^{-1} 5 p^{\prime}$ and $1 s^{-1} 6 p^{\prime}$ resonances (see Table I). The contribution of the decay channel involving a shake-down process, where the excited electron, initially promoted to the $7 p$ orbital, ends up in the $6 p$ orbital in the final state, is relatively weak (thin dashed green line). However, the interference cross term between the spectator decay and the decay through a shakedown process is rather intense (thin dash-dotted green line), giving rise to the shoulder in the cross section of the final state at the relative energy of $-0.5 \mathrm{eV}$, near the $1 s^{-1} 7 p^{\prime}$ resonance. A shoulder observed in the cross section above the IP is due to interference with the states in the continuum, which is taken into account in our fitting procedure by an arctangent function.

The preceding discussion elucidates the relative contributions of different decay channels contributing to a cross section of a given final state. Further analysis allows obtaining relative probabilities of possible decay channels for a given intermediate excited state directly from the fit parameters $k$ summarized in Table II and the peak intensities of the cross sections of the final states $I_{n p}(4 \leqslant n \leqslant 7)$. For example, for the intermediate state $1 s^{-1} 4 p^{\prime}$ decaying predominantly into the $2 p^{-2} 4 p^{+}$and $2 p^{-2} 5 p^{+}$final states, we obtain

$$
\begin{aligned}
\frac{I_{4 p}}{I_{4 p}+I_{5 p}}\left|\left\langle 4 p^{\prime} \mid 4 p^{+}\right\rangle\right|^{2}+\frac{I_{5 p}}{I_{4 p}+I_{5 p}}\left|\left\langle 4 p^{\prime} \mid 5 p^{+}\right\rangle\right|^{2} & =1, \\
\frac{I_{5 p}\left|\left\langle 1 s^{-1} 4 p^{\prime}|\mathcal{D}| \Phi_{0}\right\rangle\right|^{2}\left|\left\langle 4 p^{\prime} \mid 5 p^{+}\right\rangle\right|^{2}}{I_{4 p}\left|\left\langle 1 s^{-1} 4 p^{\prime}|\mathcal{D}| \Phi_{0}\right\rangle\right|^{2}\left|\left\langle 4 p^{\prime} \mid 4 p^{+}\right\rangle\right|^{2}} & =\alpha^{2}, \\
\frac{I_{4 p}}{I_{4 p}+I_{5 p}}\left(\alpha^{2}+1\right)\left|\left\langle 4 p^{\prime} \mid 4 p^{+}\right\rangle\right|^{2} & =1 .
\end{aligned}
$$


TABLE III. Probabilities of different decay channels for the $1 s^{-1} m p^{\prime}(m=4,5,6)$ intermediate states.

\begin{tabular}{lcc}
\hline \hline Intermediate states & Final states & Decay probability \\
\hline \multirow{2}{*}{$1 s^{-1} 4 p^{\prime}$} & $2 p^{-2} 4 p^{+}$ & 0.75 \\
& $2 p^{-2} 5 p^{+}$ & 0.25 \\
& $2 p^{-2} 4 p^{+}$ & 0.06 \\
$1 s^{-1} 5 p^{\prime}$ & $2 p^{-2} 5 p^{+}$ & 0.22 \\
& $2 p^{-2} 6 p^{+}$ & 0.68 \\
& $2 p^{-2} 7 p^{+}$ & 0.04 \\
& $2 p^{-2} 4 p^{+}$ & 0.05 \\
$1 s^{-1} 6 p^{\prime}$ & $2 p^{-2} 5 p^{+}$ & 0.16 \\
& $2 p^{-2} 6 p^{+}$ & 0.76 \\
& $2 p^{-2} 7 p^{+}$ & 0.03 \\
\hline \hline
\end{tabular}

Then the probability of the spectator decay is $\frac{I_{4 p}}{I_{4 p}+I_{5 p}}\left|\left\langle 4 p^{\prime} \mid 4 p^{+}\right\rangle\right|^{2}=\frac{1}{1+\alpha^{2}}$ and the probability of a decay through a shake-up process is $\frac{I_{5 p}}{I_{4 p}+I_{5 p}}\left|\left\langle 4 p^{\prime} \mid 5 p^{+}\right\rangle\right|^{2}=1-\frac{1}{1+\alpha^{2}}$.

The probabilities of different decay channels for the $1 s^{-1} m p^{\prime} \quad(m=4,5,6)$ intermediate states are shown in Table III. For all intermediate states the decay to the $2 p^{-2} n p^{+}$ final states with $n \geqslant 8$ has been neglected due to insufficient statistics of the data. For the same reason the decay of the $1 s^{-1} 4 p^{\prime}$ intermediate state has been limited by the $2 p^{-2} 4 p^{+}$ and $2 p^{-2} 5 p^{+}$final states and the case of the $1 s^{-1} 7 p^{\prime}$ intermediate state has been omitted. The results presented in Table III show that the decay through a shake process has a substantial probability, comparable to that of the spectator decay, and may even occur to be the dominant decay channel as is the case for the $1 s^{-1} 5 p^{\prime}$ state decaying predominantly through a shake-up process to the $2 p^{-2} 6 p^{+}$final state.

As we have shown, the analysis of our fitting results using Eq. (7) allows extracting the probabilities of different decay channels $\left|\left\langle m p^{\prime} \mid n p^{+}\right\rangle\right|^{2}$ described by the overlap integrals independently of the dipole matrix elements. Likewise, the intensities of the absorption electronic transitions $\left|\left\langle\Phi_{0}|\mathcal{D}| 1 s^{-1} m p^{\prime}\right\rangle\right|^{2}$ described by the dipole matrix elements can be obtained from the results of our fitting procedure independently of the overlap integrals. For example, for the $1 s^{-1} 4 p^{\prime}$ intermediate state decaying predominantly into the $2 p^{-2} 4 p^{+}$and $2 p^{-2} 5 p^{+}$final states, we obtain from the fit parameters presented in Table II:

$$
\begin{aligned}
\frac{I_{4 p}}{I_{4 p}+I_{5 p}} & k_{4 p}^{2}+\frac{I_{5 p}}{I_{4 p}+I_{5 p}} k_{5 p}^{2}=\left|\left\langle 1 s^{-1} 4 p^{\prime}|\mathcal{D}| \Phi_{0}\right\rangle\right|^{2} \\
& \times\left(\frac{I_{4 p}\left|\left\langle 4 p^{\prime} \mid 4 p^{+}\right\rangle\right|^{2}+I_{5 p}\left|\left\langle 4 p^{\prime} \mid 5 p^{+}\right\rangle\right|^{2}}{I_{4 p}+I_{5 p}}\right),
\end{aligned}
$$

where $k_{4 p}^{2}=\left|\left\langle 1 s^{-1} 4 p^{\prime}|\mathcal{D}| \Phi_{0}\right\rangle\right|^{2}\left|\left\langle 4 p^{\prime} \mid 4 p^{+}\right\rangle\right|^{2} \quad$ and $\quad k_{5 p}^{2}=$ $\left|\left\langle 1 s^{-1} 4 p^{\prime}|\mathcal{D}| \Phi_{0}\right\rangle\right|^{2}\left|\left\langle 4 p^{\prime} \mid 5 p^{+}\right\rangle\right|^{2}$.

Taking into account Eq. (7), we obtain

$$
\left|\left\langle 1 s^{-1} 4 p^{\prime}|\mathcal{D}| \Phi_{0}\right\rangle\right|^{2}=\frac{I_{4 p}}{I_{4 p}+I_{5 p}} k_{4 p}^{2}+\frac{I_{5 p}}{I_{4 p}+I_{5 p}} k_{5 p}^{2} .
$$

Alternatively, the dipole matrix elements can be extracted from the fit of the absorption spectrum. However, the major advantage of our method consists in the high-resolution measurements provided by the Auger spectroscopy performed in

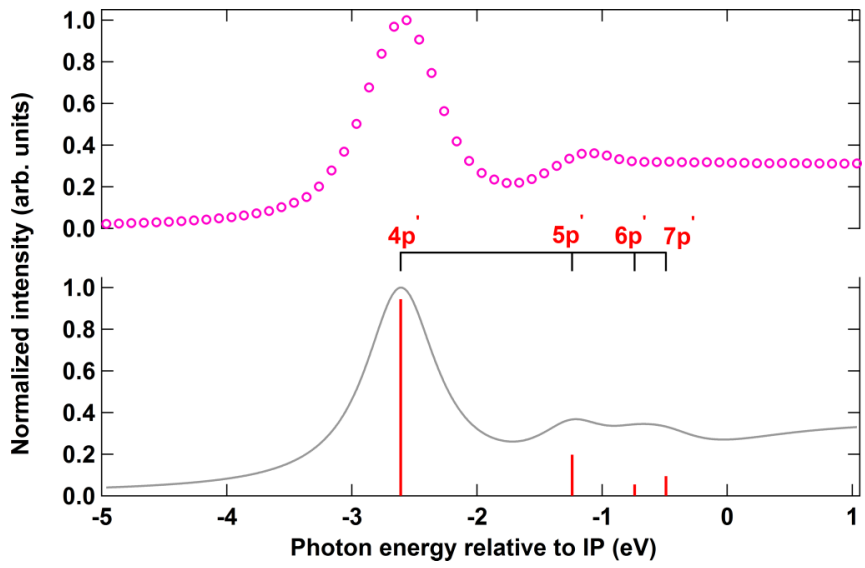

FIG. 3. Experimental (top curve) and reconstructed (bottom curve) PEY. The bars under the reconstructed PEY indicate the intensities of the $1 s-m p$ absorption transitions $(4 \leqslant m \leqslant 7)$, extracted from the fitting procedure; the solid line is the sum of the discrete contributions and the continuum contribution fitted with an arctangent function.

the Raman regime. This allows identification of the resonantly excited intermediate states summarized in Table I, which cannot be resolved in the absorption spectrum.

Generally, one can use the intensities of the absorption transitions obtained from Eqs. (8) and (9) for reconstruction of the absorption spectrum. Since our aforementioned experimental conditions do not allow measurements of the absolute cross sections, the spectrum reconstructed from the dipole matrix elements using Eqs. (8) and (9) represents a partial electron yield (PEY). The reconstructed PEY can be compared to the experimental PEY obtained directly from the measurements by integrating over the electron kinetic energy the Auger spectra recorded for different photon energies.

There is generally good agreement between the experimental and the reconstructed PEY shown, respectively, in the top and the bottom curves of Fig. 3. The bars under the reconstructed PEY indicate the intensities $\left|\left\langle\Phi_{0}|\mathcal{D}| 1 s^{-1} m p^{\prime}\right\rangle\right|^{2}$ of the $1 s-m p$ absorption transitions $(4 \leqslant m \leqslant 7)$, extracted from the fitting procedure. One can observe a slight discrepancy between the experimental and the reconstructed PEY beyond the $1 s^{-1} 7 p^{\prime}$ resonance. This is related to the fact that the intensity of the transition to the $1 s^{-1} 7 p^{\prime}$ state is overestimated, since it also takes into account the contributions of the transitions to the higher Rydberg states $1 s^{-1} m p^{\prime}$ with $m \geqslant 8$, which are otherwise neglected in the fitting procedure. The reconstructed PEY shown with a solid line includes the contributions of the transitions to the discrete states as well as the continuum contribution, which has been fitted with an arctangent function for the lack of the experimental data on the $1 s$ ionization cross section.

The reconstructed PEY shown in Fig. 3 is normalized to the intensity $\left|\left\langle\Phi_{0}|\mathcal{D}| 1 s^{-1} 4 p^{\prime}\right\rangle\right|^{2}$ of the transition to the $1 s^{-1} 4 p^{\prime}$ electronic state. The relative intensities of the transitions to the other intermediate states are $0.21 \pm 0.01$ for the $1 s^{-1} 5 p^{\prime}$, $0.06 \pm 0.01$ for the $1 s^{-1} 6 p^{\prime}$, and $0.1 \pm 0.01$ for the $1 s^{-1} 7 p^{\prime}$ electronic states as obtained from the results of our fitting procedure. 


\section{CONCLUSION}

We have demonstrated that in the hard-X-ray regime, ELI is a general phenomenon due to large lifetime broadenings of intermediate electronic states. We have shown that for a system with well separated electronic final states, our model developed for the analysis of ELI effects provides access to the fundamental observables describing excitation and decay of deep core shells. For the excitation process, the model allows extracting the intensities of the absorption transitions to the resonant intermediate states. For the relaxation process, we are able to determine the probabilities of different decay channels including spectator Auger and the decay involving shake-up and shake-down processes. Our results demonstrate a high relevance of the developed model for the interpretation of the Auger electron spectra in the hard-x-ray regime, where the data analysis inevitably faces the complexity of the interference phenomenon.

\section{ACKNOWLEDGMENTS}

Experiments were performed on the GALAXIES beamline at SOLEIL Synchrotron, France (Proposal No. 20120122). We are grateful to D. Prieur for technical assistance and to SOLEIL staff for smoothly running the facility. I.I. acknowledges financial support from Labex Plas@Par. B.C.deM. acknowledges financial support from ANR SUMMIT.
[1] T. Young, Philos. Trans. R. Soc. London 92, 12 (1802).

[2] P. Grangier, G. Roger, and A. Aspect, Europhys. Lett. 1, 173 (1986).

[3] P. Grangier and I. Abram, Phys. World 16, 31 (2003).

[4] B. Lounis and M. Orrit, Rep. Prog. Phys. 68, 1129 (2005).

[5] M. Neeb, J.-E. Rubensson, M. Biermann, and W. Eberhardt, J. Electron Spectrosc. Relat. Phenom. 67, 261 (1994).

[6] S. J. Osborne, A. Ausmees, S. Svensson, A. Kivimäki, O. P. Sairanen, A. Naves de Brito, H. Aksela, and S. Aksela, J. Chem. Phys. 102, 7317 (1995).

[7] M. N. Piancastelli, M. Neeb, A. Kivimäki, B. Kempgens, H. M. Köppe, K. Maier, and A. M. Bradshaw, Phys. Rev. Lett. 77, 4302 (1996).

[8] S. Sundin, F. K. Gel'mukhanov, H. Ågren, S. J. Osborne, A. Kikas, O. Björneholm, A. Ausmees, and S. Svensson, Phys. Rev. Lett. 79, 1451 (1997).

[9] V. Carravetta, F. Kh. Gel'mukhanov, H. Ågren, S. Sundin, S. J. Osborne, A. Naves de Brito, O. Björneholm, A. Ausmees, and S. Svensson, Phys. Rev. A 56, 4665 (1997).

[10] S. Sundin, F. Gel'mukhanov, S. J. Osborne, O. Björneholm, A. Ausmees, A. Kikas, S. L. Sorensen, A. Naves de Brito, R. R. T. Marinho, S. Svensson, and H. Ågren, J. Phys. B 30, 4267 (1997).

[11] M. N. Piancastelli, M. Neeb, A. Kivimäki, B. Kempgens, H. M. Köppe, K. Maier, A. M. Bradshaw, and R. F. Fink, J. Phys. B 30, 5677 (1997).

[12] M. N. Piancastelli, A. Kivimäki, B. Kempgens, M. Neeb, K. Maier, U. Hergenhahn, A. Rüdel, and A. M. Bradshaw, J. Electron Spectrosc. Relat. Phenom. 98-99, 111 (1999).

[13] A. Kivimäki, B. Kempgens, M. N. Piancastelli, M. Neeb, K. Maier, A. Rüdel, U. Hergenhahn, and A. M. Bradshaw, J. Electron Spectrosc. Relat. Phenom. 93, 81 (1998).

[14] U. Fano, Phys. Rev. 124, 1866 (1961).

[15] T. Åberg, Phys. Scr. T41, 71 (1992).

[16] A. Cesar and H. Ågren, Phys. Rev. A 45, 2833 (1992).

[17] J. C. Levin, C. Biedermann, N. Keller, L. Liljeby, C.-S. O, R. T. Short, I. A. Sellin, and D. W. Lindle, Phys. Rev. Lett. 65, 988 (1990).
[18] J.-E. Rubensson, M. Neeb, A. Bringer, M. Biermann, and W. Eberhardt, Chem. Phys. Lett. 257, 447 (1996).

[19] T. LeBrun, S. H. Southworth, G. B. Armen, M. A. MacDonald, and Y. Azuma, Phys. Rev. A 60, 4667 (1999).

[20] M. Kavčič, M. Žitnik, K. Bučar, A. Mihelič, S. Carniato, L. Journel, R. Guillemin, and M. Simon, Phys. Rev. Lett. 105, 113004 (2010).

[21] D. Céolin, T. Marchenko, R. Guillemin, L. Journel, R. K. Kushawaha, S. Carniato, S.-M. Huttula, J.-P. Rueff, G. B. Armen, M. N. Piancastelli, and M. Simon, Phys. Rev. A 91, 022502 (2015).

[22] R. K. Kushawaha, K. Jänkälä, T. Marchenko, G. Goldsztejn, R. Guillemin, L. Journel, D. Céolin, J.-P. Rueff, A. F. Lago, R. Püttner, M. N. Piancastelli, and M. Simon, Phys. Rev. A 92, 013427 (2015).

[23] G. Goldsztejn, T. Marchenko, D. Céolin, L. Journel, R. Guillemin, J.-P. Rueff, R. K. Kushawaha, R. Püttner, M. N. Piancastelli, and M. Simon, Phys. Chem. Chem. Phys. 18, 15133 (2016).

[24] D. Céolin, J. M. Ablett, D. Prieur, T. Moreno, J.-P. Rueff, T. Marchenko, L. Journel, R. Guillemin, B. Pilette, T. Marin, and M. Simon, J. Electron Spectrosc. Relat. Phenom. 190, 188 (2013).

[25] J.-P. Rueff, J. M. Ablett, D. Céolin, D. Prieur, T. Moreno, V. Balédent, B. Lassalle, J. E. Rault, M. Simon, and A. Shukla, J. Synchrotron Radiat. 22, 175 (2015).

[26] G. Goldsztejn, T. Marchenko, R. Püttner, L. Journel, R. Guillemin, S. Carniato, P. Selles, O. Travnikova, D. Céolin, A. F. Lago, R. Feifel, P. Lablanquie, M. N. Piancastelli, F. Penent, and M. Simon, Phys. Rev. Lett. 117, 133001 (2016).

[27] H. A. Kramers and W. Heisenberg, Z. Phys. 31, 681 (1925).

[28] G. B. Armen, J. C. Levin, and I. A. Sellin, Phys. Rev. A 53, 772 (1996).

[29] J. L. Campbell and T. Papp, At. Data Nucl. Data Tables 77, 1 (2001)

[30] E. Kukk, H. Aksela, A. Kivimäki, J. Jauhiainen, E. Nõmmiste, and S. Aksela, Phys. Rev. A 56, 1481 (1997). 